\title{
Open and Collaborative Learning Model Based on Metacognitive Strategies
}

\author{
Liviana TUDOR ${ }^{1}$, Adrian MOISE ${ }^{2}$ \\ ${ }^{1}$ Dept. of Informatics, Information Technology, Mathematics and Physics, \\ Petroleum - Gas University of Ploiesti \\ 39, Bd. Bucuresti, 100680, Ploiesti, Romania \\ LTudor@upg-ploiesti.ro \\ ${ }^{2}$ Dept. of Automatic Control, Computers and Electronics, \\ Petroleum - Gas University of Ploiesti, \\ Bd. Bucuresti Nr. 39, 100680, Ploiesti, Romania \\ AMoise@upg-ploiesti.ro
}

\begin{abstract}
In this paper, the authors describe an open and collaborative model based on metacognitive neural strategies applied to students' research projects in Artificial Neural Networks. The model is focused both on instructor-student and student-student interactions and includes an on-line knowledge-based intelligent system as well as several social psychological determinants. The performance of the proposed model is proved by the results obtained by the MSc students, their motivation to complete a postgraduate course and it is justified by the highly useful online knowledge base. The functionality and efficiency of the intelligent system are highlighted by traffic and log analysis of the Open Source data collections used in the disciplines which have been involved in the experiment.
\end{abstract}

Keywords: open and collaborative learning model, metacognitive strategies, artificial neural networks, online knowledge based intelligent system, dissertation works.

\section{Introduction}

The significant progress in Information Technology in the last years has imposed a steady training process of students in Artificial Intelligence and has also required a certain adaptation of the university curriculum in order to integrate knowledge about automatic learning and machine learning [1]. Automatic learning studies systems that are able to improve their performance based on a set of training data [2].

The academic curriculum for MSc studies in Informatics and Systems Engineering could be a component of the student-centred curricula. A curriculum is a set of teaching, learning and evaluation competences that allows the university to offer the students a whole system of information, skills, behaviours and competencies. One of the optional subjects in a university, for Informatics MSc level, allows the development of skills in the Artificial Neural Networks (ANN) field and belongs to the Artificial Intelligence curricula area. The status of the ANN as an elective field of study means it could be studied, at the students' request, bringing their needs and interests to the foreground.

ANN teaching and learning should be based on an Open and Collaborative Learning Model
(OCLM) and on interactive teaching methods. Collaborative learning of neural algorithms has as a major goal the training of students so they can acquire interdisciplinary skills and carry out team research projects, in a cooperation and competition framework. The instructor student collaboration is based on developing and using neural metacognitive strategies. The metacognitive action, the thought about thought, represents a high-level cognitive activity. When it is related to the learning activity, the metacognitive competence defines a measure of: what a student knows, what a student knows he doesn't know, the knowledge and perception about how learning occurs and the manner to make the learning process efficient [3].

The original contribution of this paper lies in the methodological and scientific aspects and is summarized below. (1) A collaborative teaching and learning method, based on scientific research projects in ANN, will be described. The impact on the efficiency of teaching activity will be studied, while using the collaborative teaching and learning method based on neural metacognitive competencies. An experimental study makes an analysis of the MSc students results over two years of study. (2) Development of an Open and Collaborative Learning Model (OCLM) can be done by building a virtual teaching, learning and 
evaluation environment; that means, a software package that combines Web technologies with Artificial Intelligence techniques applied to education. The adaptation process of the OCLM to the MSc students' expectations is done by developing intelligent systems based on online knowledge.

The paper is structured as follows. In the section titled Related works the authors present the main research results in using Artificial Intelligence methods and techniques for teaching and learning activities and how this paper is related to the already known literature. The section titled Design of open and collaborative learning describes the open and collaborative learning steps as well as the scaffolding strategies based on specific questions technique. The section titled Developing metacognitive neural strategies, describes a method to develop metacognitive neural strategies for ANN research projects. The section titled Developing an open and collaborative learning model shows the OCLM architecture and its components. The experimental study, the OCLM performance analysis as well as the results of testing the online knowledge-based intelligent system are all shown in the section titled A pedagogical experiment with OCLM. The Conclusions section summarizes the main contributions of the study.

\section{Related Works}

Over the last decades, research in the field of Artificial Intelligence applied to Education has brought to the fore Intelligent Learning Environments (ILE), Intelligent Tutoring Systems (ITS), Managed Learning Environments [4], Cognitive Tools, Open Learner Models (OLM), Project-Based Learning (PBL) and other hybrid techniques that can be applied in teaching, learning and evaluation activities [1]. Most ITS use expert system technology and are based on research on educational knowledge representation and corresponding data models [5]. Some studies define ILE as a combination of an ITS and a learning environment that allows for studentdriven learning [6]. Some experts suggest that the appropriate role for a computer is that of a mind-extension cognitive tool [7]. Cognitive tools are not intelligent tools, because planning, decision-making, and self-regulation are the responsibility of the learner, not the technology.
OLM are usually integrated into an adaptive learning environment for consultation by the learner during their interaction [8]. Many OLM use skill meters for display, problem solving and other tutorial support in an intelligent tutoring system [9] and in adaptive educational hypermedia providing navigation support [10]. PBL offers many advantages and challenges when implemented in the classroom: increased motivation, increased problem solving ability, improved media research skills, increased collaboration, increase resource management skills and many others [11]. During the last decade, free e-learning platforms such as Moodle, ATutor, LogiCampus have been used. Also, recommendation systems have been developed and dedicated to e-learning environments which are useful in SelfRegulated Learning and use data mining and classification algorithms [12], [13].

Taking into consideration the learning methods presented above, this paper will introduce an Open and Collaborative Learning Model for the academic environment. OCLM is based on metacognitive strategies used in scientific research projects and it is virtually implemented using Artificial Intelligence techniques as well as online knowledge based systems.

\section{Design of Open and Collaborative Learning}

Learning in a collaborative environment means learning in a group. Empirical research [14] confirm the hypothesis that solving tasks in a working group requires activating higher cognitive processes that occur with difficulty in the individual learning type.

A collaborative environment enables students to access a common knowledge kernel, putting them in a position to consume information, and to produce knowledge and to negotiate its meanings [15]. Research that have tried to develop this theory reveals cognitive features and benefits of the types of interactions that are characteristic for a group dynamics [16]. The learning social theory states that learning is, essentially, related to participation.

The principle according to which learning tasks are planned by the instructor is mentioned in the general theory of collaborative learning. During the learning process, each step in solving tasks has a leader and the leader can be the instructor, any group member or any group. 
The steps in solving learning tasks in a collaborative manner can be described as follows [3]: (1) The instructor assigns tasks; (2) Each working group member explains the individual understanding of the learning tasks; (3) The working group (helped by the instructor, if necessary) negotiates common meaning of learning tasks; (4) The working group identifies strategies to solving tasks; (5) The working group solves the assigned tasks.

The authors of this paper propose an open and collaborative learning model that can be applied in academic environments, a model in which instructors and students interactively take part in choosing curriculum and establishing learning tasks.

\subsection{Scaffolding strategies for metacognitive competencies developing}

Open and collaborative learning in a university, at the MSc level, can be done by using study groups, team working for research projects, and it is based on student - student and student instructor interactions.

In order to systematically address the open and collaborative learning tasks setting, student should be supported by a metacognitive scaffolding [17]. Most students do not show a metacognitive self-determined behavior and that's why it is necessary to integrate the metacognitive scaffolding into the training strategy. Metacognitive scaffolding role is to help students to form and adopt a selfdetermined reflective behavior in learning.

Management skills in educational context can be developed by using metacognitive strategies that include: learning and research planning strategies, monitoring and self-control strategies, and evaluation strategies [18]. In order to form and develop metacognitive competencies in a collaborative learning environment, the instructor has to provide metacognitive scaffolding methods and instruments such as: strategic questions method, the log technique [3]. For this study, the authors have chosen and adapted a metacognitive scaffolding method which is appropriate for open and collaborative learning. The method itself uses the strategic questions technique. A list of customized questions will be presented for three types of strategies that correspond to research projects based learning; projects are implemented by students teams.
According to the existing educational theories, Project-Based Learning (PBL) can be divided into three main processes [11]. In the planning phase, the learner chooses the topic, searches for resources for needed information, and organizes the resources. In the implementation phase, the learner develops the project idea, combines the contributions of the group, and builds the project. In the processing phase, the project is shared with other groups, and feedback is obtained [11].

The scaffolding strategies are adapted to the research projects based open and collaborative learning: (a) planning phase, (b) implementation and monitoring phase, and (c) processing and evaluation phase.

\section{Developing Metacognitive Strategies}

Teaching MSc students includes an important research plan that can be performed through case studies, research projects and dissertation works. Metacognitive strategies can be developed by instructor-student and studentteam collaboration during the solving stages of team research projects.

In this section a way to developing neural metacognitive strategies will be presented; these strategies are required by the research activity of MSc students, for projects in the ANN field. The neural metacognitive skills aim at developing the ability to identify problem in various fields (problems that can be solved by using connectionist computing properties) as well as to know the specific characteristics of neural model: calculations are performed in parallel and a learning process replaces an a priori development program [2].

A way to develop neural metacognitive strategies will be shown in details; the specific questions method will be used and the three stages will be covered.

(a) Planning a research project means setting up specific tasks, solving stages and, in general, finding answers to all the specific questions of the planning phase.

What tasks should be accomplished in an ANN research project? The answer to this question should start with the first task: understanding the definition of an ANN. An ANN is a structure composed by a certain number of interconnected elementary computation units (artificial neurons). Each neuron has an input- 
output (I/O) characteristic, can have connections to other neurons and implements a local computation process or a function [2]. Other tasks of the project are: explaining the difference between an ANN and an usual processor, listing advantages and disadvantages of ANN, understanding the systemic of the network output, finding and consulting the references that have to be used in solving the project, and finally establishing the solving stages of a specific ANN problem (for example, recognizing decimal digits written in a 10 pixels $x 8$ pixels area). What kind of knowledge is needed for the assigned task to be performed? For the above mentioned problem, MSc students should have knowledge in Calculus, Matlab, matrix calculation and graphic interpretation of results. What is the task goal? The goal is to obtain a viable network for solving the given problem. An ANN functionality depends on how the input signals are processed and transformed into output signals, by using the neurons' characteristics, the network topology and a learning algorithm [19]. The learning objective is to get a network with a chosen architecture and to find the interconnection weights [2].

What is the solving plan? In solving an ANN problem, the student should touch the stages briefly sketched further.

1. Setting up the network inputs. The inputs have to be quantifiable and to be found by using a measurement process. Furthermore, possible deformations of invariant properties of input patterns have to be considered as well as noise sources.

2. Setting up the network outputs. These signals have to be understood and translated by the user into real meanings.

3. Setting up the network architecture or topology. The interconnection type (recurrent or non-recurrent), number of layers, number of neurons on each layer have to be established.

4. Choosing the neurons' characteristic. If a biased input is used, the network offers more flexibility in performing computing operations.

5. Choosing a learning (training) algorithm. For example, Perceptron Learning, Delta Rule, Generalized Delta Rule, Neural Associator, Backpropagation could be used [2].

6. Setting up the training set, a collection of pairs (known input vector, desired output).
It is highly recommended to use also input vectors that do not represent ideal values; that is, students should use disturbed input patterns when establishing the training set.

7. Applying the training algorithm with the chosen training set. As a result, the interconnection weights are obtained.

8. Simulating the network. The user applies many testing sets in order to verify the network viability.

9. If the network is not viable, the network architecture and/or parameters are changed and the whole process is resumed.

(b) Implementation, monitoring and selfcontrol stages concerning an ANN project can have a few guidelines. Network topology and neurons' characteristics are set up based on a rigorous scientific justification and the training algorithm is applied. The whole team should participate in brainstorming meetings to establish the training set and testing sets, to comment the final results and to establish real ways to improve the project performance.

(c) The processing and evaluation strategies for the research project are based on a few rules. After the project has been successfully tested, the results should be presented in text, tables, graphs etc. Also, conclusions, strengths and weaknesses, and suggestions for future developments are presented and sustained.

Then, the initial goals, methods, steps taken and comparative results obtained with different methods are presented in front of an audience.

\section{Developing an OCLM}

OCLM based on scientific research projects is suitable and applicable in academic environments, for MSc studies, and complements modern educational techniques because it stimulates individual creativity and performance. It also develops metacognitive skills in managing and evaluating one's own work. The social and psychological component of OCLM allows the creation of collaborative social skills for team working and fulfilment of communication psychological needs.

\subsection{OCLM architecture}

Developing a student-centered OCLM means creating and interactively using a virtual environment based on Web technologies and Artificial Intelligence techniques. OCLM 


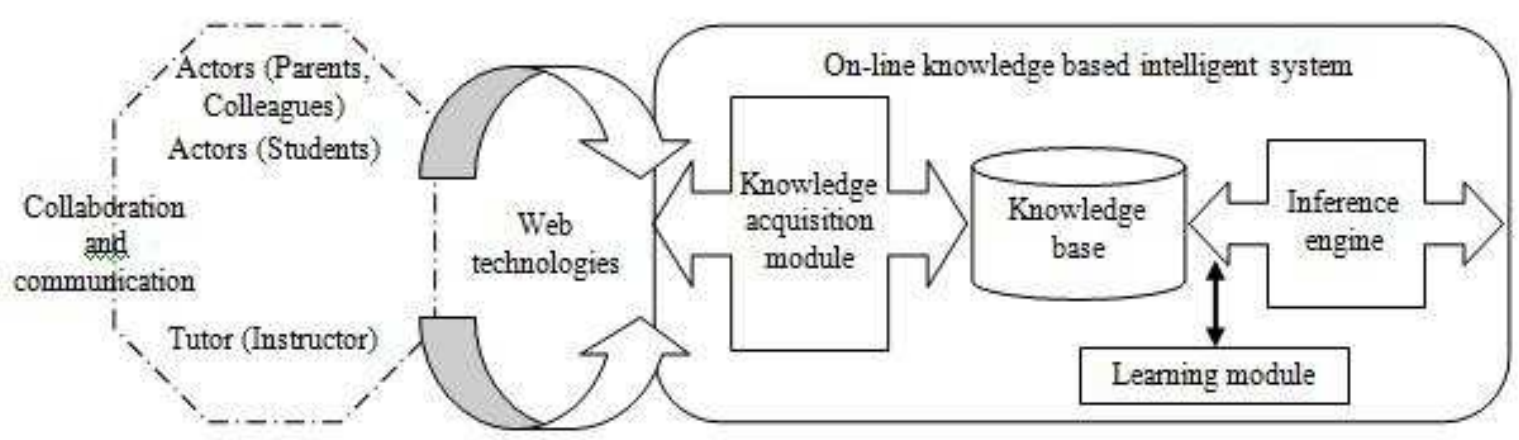

Figure 1. OCLM architecture

includes an on-line knowledge-based intelligent system as well as a social and psychological component (Figure 1).

The main components of the on-line knowledge based intelligent system are: the knowledge base, the inference engine, the knowledge acquisition module, the interface and the learning module. The on-line system interface interacts with the Webmaster and the Web users which develop and manage the knowledge basis, query the knowledge base and present the required results. The knowledge acquisition module integrates the new knowledge and interacts with the user interface and the knowledge base. Knowledge acquisition can be done directly, from the environment, or by developing new knowledge through the intelligent system interface. In general, a knowledge base contains Horn clauses that can be represented by facts and rules. Facts are static knowledge and rules express dynamic knowledge or actions rules [20]. If this concept is applied to the on-line knowledge based intelligent system, the knowledge base includes: (a) Open Source data collections, (b) educational instruments, and (c) rules for knowledge processing. The inference engine activates the knowledge base rules and derives new knowledge [20]. The Open Source data collections contain teaching materials that belong to Computer Science: Artificial Neural Networks, Expert Systems, Databases, Computer Programming etc. Educational resources can be accessed by instructors and students via the files that contain courses, evaluation tests, curricula, dissertation subjects, activities planning etc.

The knowledge-based system becomes intelligent through the learning module since this module allows enhancing system performances by deriving new knowledge from the existing ones [19]. Learning is a characteristic of intelligent systems and it represents the cognitive process of acquiring knowledge and skills [12]. The learning module uses memorized knowledge about the problem solution.

\subsection{The social and psychological module}

OCLM includes a social and psychological component that develops the social theory of active participation, in groups, in the learning process, by: communication, collaboration, and adaptation. Collaboration inside a team has psychological benefits because the students are asked to have an active role in teaching, learning and evaluation. In turn, the instructor enhances students' self-confidence, adapting capacities, and responsibility for the own activity. When the OCLM is applied to ANN, collaboration and communication instructor student are focused on developing metacognitive neural strategies. Working in teams for solving research neural projects will make students learn how to cooperate, to discover and process information, to set-up connexions between neural theoretical concepts and real life problems.

\subsection{Software implementation method}

OCLM uses an e-Learning platform based on Open source data collections and the educational Moodle software stored at http://www.programare.biz/E/indexE.html and http://programare.biz/moodle/login/index.php.

The learning module is revealed by on-line evaluation tests that include memorized questions and answers in the system knowledge database. An online evaluation test example realized in Javascript and $\mathrm{Html}$ is available at www.programare.biz/E/RNAE/TRNAe.htm. 


\section{A Pedagogical Experiment with OCLM}

The open and collaborative method based on metacognitive neural strategies development for research projects has been analysed for the discipline Artificial Neural Networks; this discipline is included in the MSc curricula for two specializations, Informatics and Advanced automation, at the authors' university. A pedagogical experiment will be developed. During this experiment, traditional methods and the proposed OCLM have been alternatively applied in teaching, learning and evaluation processes.

Then, a comparative analysis has to be done to highlight students' motivation and results when dissertation works are developed. These works have been coordinated by instructors involved into the experiment. The experiment has been performed by using two samples (groups of students): the control group (witness) and the experimental group. During the university year 2013 - 2014, instructors involved into the pedagogical experiment chose classical teaching methods for the control group, while in $2014-2015$, they used the OCLM for the experimental group.

\subsection{OCLM performance analysis}

A conclusive analysis of the OCLM can be done by taking into consideration the following parameters: (a) the number of students who choose to make a dissertation work in ANN, (b) the number of students who choose to make dissertation works in all the disciplines taught by the instructors involved into the experiment, (c) the number of students who obtained a pass grade on a dissertation in ANN, and (d) the number of students who obtained a pass grade on a dissertation in all the disciplines taught by the instructors involved into the experiment.

Figure 2 reveals superior results for the experimental group, for which the OCLM was used, in comparison with the control group, for which the classical teaching and learning methods were used. The number of students who completed master studies with a dissertation work in ANN (parameter c), for the experimental group, was higher than the same parameter for the control group. Similarly, the number of dissertation works in all the disciplines (parameter $\mathrm{d}$ ), for the experimental group, was higher than the same parameter for the control group.

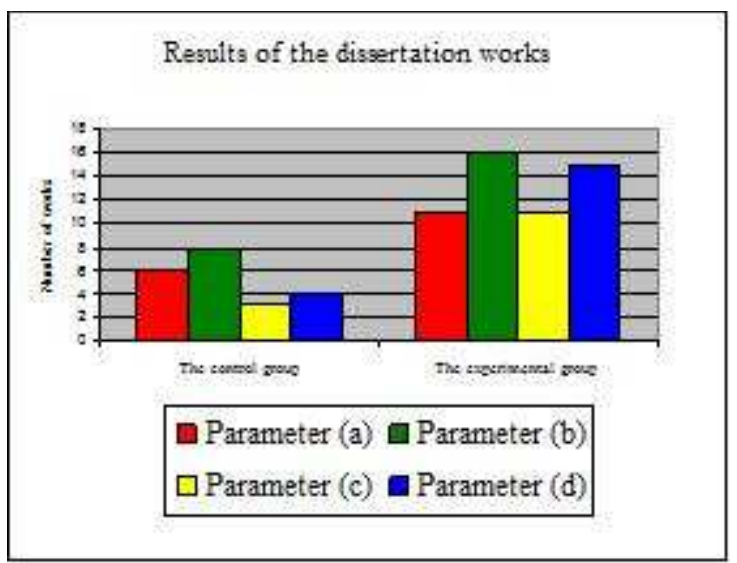

Figure 2. Comparative analysis of the results of groups of students

To investigate the efficiency of the proposed OCLM, two functions have been defined to evaluate the degree in which MSC studies were completed (both for ANN dissertations and all disciplines dissertations), for the $n$ instructors involved into the pedagogical experiment. Then

$$
\operatorname{Deg}_{A N N}(n)=\sum_{i=1}^{n} C_{A N N}(i) \text {, }
$$

where $C_{A N N}(i)$ represents the MSc graduation coefficient with ANN dissertation works, coordinated by the instructor $i$. This coefficient can be found (on a 0 to 1 scale) as a ratio between the number of ANN dissertation works presented and the number of ANN dissertation works assigned. Similarly,

$$
\operatorname{Deg}_{T}(n)=\sum_{i=1}^{n} C_{T}(i) \text {, }
$$

where $C_{T}(i)$ is the MSc graduation coefficient with all the disciplines dissertation works, coordinated by the instructor $i$.

Students' motivation to complete MSc studies can be found, for each group of students, as an average of graduation degree in ANN (1) and that in all the other disciplines (2):

$$
M(n)=\left(\operatorname{Deg}_{A N N}(n)+\operatorname{Deg}_{T}(n) / 2\right.
$$

In Table 1 the MSc graduation coefficients and students' motivation are presented. They have been determined by applying equations (1), (2) and (3). MSc graduation degree for the two groups are shown in Figure 3 and the graphical representation proves the efficiency of applying the OCLM. Students in the experimental group, who completed MSc in Informatics or System Engineering by presenting ANN dissertation 
works or any other discipline included into the OCLM based pedagogical experiment, had better results than the students in the control group, for which traditional education methods were used. Also, students' motivation to complete MSc studies was higher for the experimental group than for the control group.

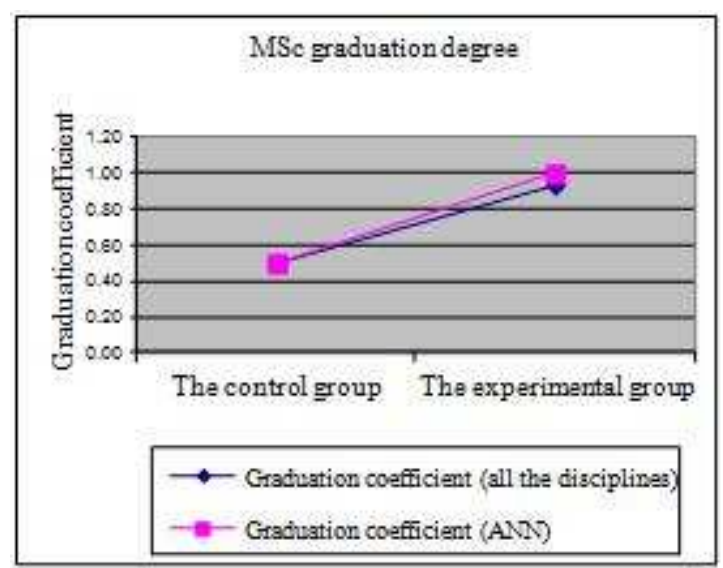

Figure 3. MSc graduation degree analysis.

\subsection{Testing the on-line knowledge-based intelligent system}

In order to test the intelligent system, a traffic analysis has been done and an analysis of the log events for the Open Source collections of the disciplines that were taught by the instructors involved into experiment. The last 8 months statistics include: HTTP (Web) use, number of visitors, number of unique IP addresses, number of accessed pages and bandwidth (Figure 4). Knowledge-based intelligent system functionality has been proved also by the place of access (countries) and the modality to access the Web pages (direct or using Web search robots such as Googlebot, Crawler etc) (Table 2 and Table 3). The above mentioned site has a Google index that is used by the search engines to rapidly find information.

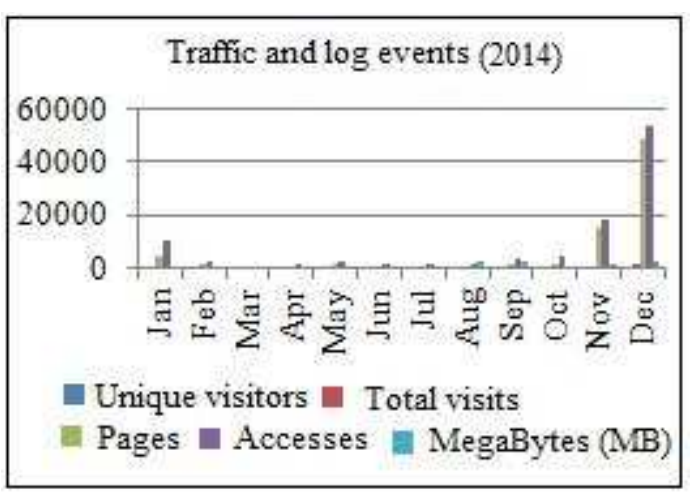

Figure 4. Traffic and log events analysis.
Table 1. MSc graduation coefficients

\begin{tabular}{|l|c|c|}
\hline $\begin{array}{l}\text { MSc graduation } \\
\text { degree }\end{array}$ & $\begin{array}{c}\text { Control } \\
\text { group }\end{array}$ & $\begin{array}{c}\text { Experimental } \\
\text { group }\end{array}$ \\
\hline $\begin{array}{l}\text { All the disciplines } \\
\text { graduation coefficient }\end{array}$ & 0.5 & 0.94 \\
\hline $\begin{array}{l}\text { ANN graduation } \\
\text { coefficient }\end{array}$ & 0.5 & 1 \\
\hline Students'motivation & 0.5 & 0.96875 \\
\hline
\end{tabular}

Table 2. Intelligent system accessing statistics.

\begin{tabular}{|c|c|c|c|}
\hline Countries & Pages & Views & Bytes \\
\hline Romania & 74,740 & 95,049 & $3.45 \mathrm{~GB}$ \\
\hline U.S.A. & 1,255 & 2,395 & $3.59 \mathrm{~GB}$ \\
\hline China & 411 & 580 & $31.66 \mathrm{MB}$ \\
\hline Italy & 250 & 353 & $42.22 \mathrm{MB}$ \\
\hline Japan & 249 & 260 & $6.45 \mathrm{MB}$ \\
\hline
\end{tabular}

Table 3. Access mode.

\begin{tabular}{|c|c|c|}
\hline $\begin{array}{c}\text { Users / Robots } \\
\mathbf{2 0 1 4}\end{array}$ & $\begin{array}{c}\text { Accesse } \\
\text { s }\end{array}$ & Bytes \\
\hline Login with users & 102,862 & $10.80 \mathrm{~GB}$ \\
\hline Googlebot & 14,526 & $605.53 \mathrm{MB}$ \\
\hline Unknown robot & 14,534 & $169.29 \mathrm{MB}$ \\
\hline Yahoo Slurp & 51 & $769.85 \mathrm{~KB}$ \\
\hline
\end{tabular}

\section{Conclusions}

This paper presents an open and collaborative learning model based on neural metacognitive strategies that are specific to research projects for MSc studies. The effectiveness of the proposed model has been proved by analyzing the MSc students results and by testing the functionality of the on-line knowledge based intelligent system that was used by the instructors involved into the pedagogical experiment.

\section{REFERENCES}

1. BISWAS, G., J. S. KINNEBREW, How Do Students' Learning Behaviors Evolve in Scaffolded Open-Ended Learning Environments?, Proceedings of the 21st International Conference on Computers in Education ICCE, Indonesia, Nov. 2013.

2. BRUSILOVSKY, P., Student Model centered Architecture for Intelligent Learning Environments, Proceedings of 
the 4th International Conference on User Modeling, Hyannis, MA, USA, Aug. 2004.

3. BULL, S., GARDNER, P, Highlighting Learning Across a Degree with an Independent Open Learner Model, Artificial Intelligence in Education, IOS Press, Amsterdam, 2009, pp. 275-282.

4. Farrell, L., Negotiating Knowledge in the Knowledge economy: Workplace educators and the politics of codification, Studies in Continuing Education, vol. 23(2), 2001, pp. 201-214.

5. HAN, S., K. BHATTACHARYA, Constructionism, Learning by Design, and Project Based Learning. in M. Orey (Ed.), 2001.

6. HURME, T. R., Metacognition In Group Problem Solving - A Quest For Socially Shared Metacognition. Juvenes Print Tampere, University of Oulu, Finland, 2001.

7. LAJOIE, S. P., Computers as Cognitive Tools. II, New Jersey, Lawrence Erlbaum Associates Inc., 2000.

8. MANASIA, L., A. PARVAN, Dezvoltarea competentei metacognitive în mediile colaborativ-virtuale de învătare, National Conference on Virtual Learning, Romania, 2013.

9. MITROVIC, A., B. MARTIN, Evaluating the Effect of Open Student Models on Self-Assessment, International Journal of Artificial Intelligence in Education, vol. 17(2), 2007, pp. 121-144.

10. MOISE A., Neural Networks for Pattern Recognition (in Romanian). MATRIX ROM Publishing House Bucharest, Romania, 2005.

11. SARYAZDI, N. G., H. MIRASHK, A. A. KARDAN, Learner Clustering and Association Rule Mining for Content Recommendation in Self-Regulated Learning, International Journal of Computer Science Research and Application, vol. 1, 2012, pp. 69-78.
12. THORSTEINSSON, G., A. NICULESCU, Examining Teachers' Mindset and Responsibilities in Using ICT, Studies in Informatics and Control, vol. 22 (3), 2013, pp. 315-322.

13. TUDOR, N. L., Neural networks. Matlab applications (in Romanian), MATRIX ROM Publishing House, Bucharest, Romania, 2012.

14. TUDOR, N. L., Logic Programming and Expert Systems. Visual Prolog and Exsys Applications (in Romanian). MATRIX ROM Publishing House Bucharest, Romania, 2012.

15. TUDOR, N. L., MOOC Projects based on Numerical Calculation Metacognitive Strategies. Romanian Journal of Information Technology and Automatic Control vol. 25(1), 2015.

16. VLADA, M., Computer Science for Future Education and ICVL Project objectives, International Journal of Computer Science Research and Application, vol. 2(1), 2012, pp. 1-4.

17. WEBER, G., P. BRUSILOVSKY, ELMART: An Adaptive Versatile System for Web-Based Instruction, International Journal of Artificial Intelligence in Education, vol. 12, 2001, pp. 351-384.

18. WENGER, E., Communities of Practice: Learning, Meaning, and Identity, Cambridge Univ. Press, New York, 1998.

19. WENGER, E., Artificial Intelligence and Tutoring Systems, Morgan Kaufmann, Los Altos, CA, 1987.

20. A WHITEBREAD D., COLTMAN, P., PASTERNAK, D.B., BINGHAM, S., ALMEQDAD, Q., DEMETRIOU, D., The Development of Two Observational Tools for Assessing Metacognition and SelfRegulated Learning in Young Children. Metacognition and Learning, 4, 2009 